\title{
Positional Specificity of Gastric Hydrolysis of Long-Chain n-3 Polyunsaturated Fatty Acids of Seal Milk Triglycerides ${ }^{1}$
}

\author{
Sara J. Iverson ${ }^{a, b, *}$, Joseph Sampugna ${ }^{b}$ and Olav T. Oftedal ${ }^{a}$ \\ ${ }^{2}$ National Zoological Park, Smithsonian Institution, Washington, D.C. 20008 and ${ }^{b}$ Department of Chemistry and Biochemistry, \\ University of Maryland, College Park, Maryland 20742
}

Long-chain n-3 polyunsaturated fatty acids (n-3 PUFA) of marine oils are important dietary components for both infants and adults, and are incorporated into milks following maternal dietary intake. However, little is known about the hydrolysis of these PUFA from milk triglycerides (TG) by lipases in suckling young. Seals, like humans, possess gastric lipase; however, the milk lipids of seals and sea lions are almost devoid of the readily hydrolyzable medium-chain fatty acids, and are characterized by a large percentage (10-30\%) of n-3 PUFA. Gastric hydrolysis of milk lipids was studied in vivo in suckling pups of three species (the California sea lion, the harp seal and the hooded seal) in order to elucidate the actions and specificity of gastric lipases on milk TG in relation to fatty acid composition and TG structure. Regardless of milk fat content (31-61\% fat) or extent of gastric hydrolysis (10-56\%), the same fatty acids were preferentially released in all three species, as determined by their relative enrichment in the free fatty acid (FFA) fraction. In addition to 16:1 and 18:0, these were the PUFA of 18 carbons and longer, except for 22:6n-3. Levels of 20:5n-3 were most notably enriched in FFA, at up to five times that found in the TG. Although 22:6n-3 was apparently also released from the TG (reduced in the diglyceride), it was also notably reduced in FFA. Positional analysis of milk TG based on the products of Grignard hydrolysis revealed that these PUFA, including 22:6n-3, were preferentially esterified at the $\alpha$-position of the TG, and that the fatty acids not released during gastric hydrolysis were located at the $8 n-2$ position. The extreme reduction of 22:6n-3 and enrichment of 20:5n-3 in FFA is discussed. Results from this study are consistent with reports that gastric lipase acts stereospecifically to release fatty acids at the $\alpha$-positions $(8 n-3$, $8 n-1)$. We conclude that the n-3 PUFA in milk are efficiently hydrolyzed by gastric lipase and that this has im. portant implications for digestion of milks enriched in PUFA by neonates in general.

Lipids 27, 870-878 (1992).

Long-chain n-3 polyunsaturated fatty acids ( $n-3$ PUFA), characteristic of marine oils, have become a major focus of research due to their potent antiatherogenic $(1,2)$,

\footnotetext{
${ }^{1}$ Based on a paper presented at the Symposium on Milk Lipids held at the AOCS Annual Meeting, Baltimore, MD, April 1990; part of this work is from the doctoral dissertation by S.J.I., University of Maryland, 1988.

*To whom correspondence should be addressed at: the Canadian Institute of Fisheries Technology, Technical University of Nova Scotia, 1360 Barrington St., P.O. Box 1000, Halifax, Nova Scotia, B3J 2X4 Canada.

Abbreviations: BHT, butylated hydroxytoluene; DG, diglyceride; FFA, free fatty acid; GC, gas chromatograph; GLC, gas-liquid chromatography; HPLC, high-performance liquid chromatography; MCFA, medium-chain fatty acid; MG, monoglyceride; MUFA, monounsaturated fatty acid; PUFA, polyunsaturated fatty acid; SFA, saturated fatty acid; TG, triglyceride; TLC, thin-layer chromatography.
}

antineoplastic, and antiinflammatory (3) effects. Recent evidence suggests their intake to be important from an early age for these reasons (i.e., eicosanoid synthesis), as well as for normal neonatal brain and nervous development and cell membrane structure (4-7). Long-chain n-3 PUFA are readily incorporated into milks of various species following maternal dietary intake $(8,9)$. However, information on the hydrolysis and absorption of these $n-3$ PUFA in milks by suckling young is limited.

Milk lipid is composed primarily of triglyceride (TG; 97-99\%) and, in order to be absorbed in the intestine, must be hydrolyzed to free fatty acid (FFA) and monoglyceride (MG). Although lipids are essential to the neonate for both normal growth and metabolism, in species that have been studied (e.g., human, rat, dog), intestinal function is immature, resulting in reduced levels of pancreatic lipase and bile salts (10-12), both of which are required for the intestinal digestion of fat. Additionally, it is reported that pancreatic lipase, which is specific for the $\alpha$-positions ( $s n-1$ and $s n-3$ ) of TG, does not hydrolyze TG ester bonds containing long-chain n-3 PUFA $(13,14)$. In fish oils, n-3 PUFA are esterified primarily at the $s n-2$ position of the TG and thus can be readily absorbed as $s n-2 \mathrm{MG}$ upon pancreatic lipase action (15-18). However, the n-3 PUFA in depot fats of mammals are located primarily at the $s n-3$ and secondarily at the $s n-1$ positions (18-21), and thus whale oil TG have been found to be resistant to pancreatic lipase (22).

Thus, in view of these relative inadequacies, fat absorption in newborns may depend upon compensatory or alternative mechanisms of hydrolysis by means of preduodenal lipases. In particular, initial intragastric lipolysis by lingual and gastric lipases may be especially important for the digestion of milk fat, which is packaged as globules and is otherwise resistant to pancreatic lipase $(23,24)$. However, studies of lipolysis by gastric and lingual lipases have been based largely on in vitro studies using a single component fatty acid in artificial lipid emulsions and have stressed their preferential release of medium-chain fatty acids (MCFA). While milks of some species contain notable proportions of MCFA, milk lipids of humans and most carnivores are composed mostly of long-chain fatty acids (25-27). Milk lipids of seals and sea lions furnish $85-95 \%$ of the total caloric intake of neonates and are characterized primarily by long-chain and unsaturated fatty acids, including a very large percentage (10-30\%) of $n-3$ PUFA derived from their marine diets $(28,29)$. Suckling pups consume phenomenal quantities of lipid daily at $600 \mathrm{~g}$ to $5 \mathrm{~kg}(30,31)$, or up to 10 - to 20 -fold higher on a body weight basis than neonates of the human or dog. Rapid blubber deposition suggests rapid and efficient absorption of milk lipid $(28,32,33)$, but the mechanisms of hydrolysis and digestion are unknown. Gastric lipase is present in seal pups (34); however, milks are virtually devoid of the readily hydrolyzable MCFA. These species thus provide an excellent opportunity to elucidate the actions of gastric lipases on milk TG in relation to fatty acid composition and TG structure. 
In this study we examined milk lipid hydrolysis by gastric lipase in suckling neonates of the harp seal (Phoca groenlandica), the hooded seal (Cystophora cristata) and the California sea lion (Zalophus californianus). Our objectives were to characterize the location of the n-3 PUFA in seal milk TG by positional analysis using Grignard hydrolysis (35) and to measure in vivo hydrolysis during intragastric digestion. We used data on the milk TG structure and comparison of the proportions and compositions of hydrolysis products, produced during both gastric lipase hydrolysis and Grignard hydrolysis, to elucidate the actions and stereospecificity of gastric lipase and the digestibility of n-3 PUFA in milk.

\section{MATERIALS AND METHODS}

Sample collection. Samples were collected from mothers and pups of harp seals and hooded seals on the pack ice off the southeast coast of Labrador, Canada, and from California sea lions on San Nicolas Island, Channel Islands, California. Milk was manually expressed from chemically immobilized females following administration of oxytocin. Prior to analysis, milk samples were stored frozen at $-20^{\circ} \mathrm{C}$ (sea lion) or frozen in chloroform/methanol $(2: 1$, $\mathrm{vol} / \mathrm{vol}$ ) containing $0.01 \%$ butylated hydroxytoluene (BHT) (harp and hooded seals) from the time of collection.

Pups were physically restrained and stomach contents (ingested milk) were evacuated by intubation with a $3 / 8$ inch veterinary stomach tube, opportunistically in harp $(n=15)$ and hooded seals $(n=17)$, or at timed intervals following observed suckling in sea lions $(n=20)$. At the time of collection, all samples were placed in chloroform/ methanol $(2: 1, \mathrm{vol} / \mathrm{vol})$ with BHT and stored frozen at $-20^{\circ} \mathrm{C}$ until analysis. Characterization of gastric hydrolysis (analysis of milk TG and hydrolysis products) was investigated in five mothers and pups of each species.

Neutral lipid class analysis and separation. All solvents used were HPLC (high-performance liquid chromatography) grade or analyzed reagent grade and all containers and equipment were composed of glass or teflon and pre-washed with solvent prior to use. Samples were extracted using a modification $(25,28)$ of the method of Folch et al. $(36)$.

Proportions of neutral lipid classes were determined in milk and gastric samples by thin-layer chromatography (TLC) and quantitative densitometry. Plates used for TLC were commercial $20 \times 20 \mathrm{~cm}$, precoated (Si250-PA) channeled glass plates (Baker Chemical Co., Phillipsburg, NJ) with a preadsorbent spotting area. Plates were predeveloped in $100 \%$ diethyl ether in order to remove the organic binder which typically runs at the solvent front, and were activated at $120^{\circ} \mathrm{C}$ for $20 \mathrm{~min}$ before spotting. A two-stage development process was employed using chloroform/ methanol/ethanol/acetic acid (98:2:1:0.1, by vol) (partial plate development to $17 \mathrm{~cm}$ ) followed by hexane/diethyl ether/acetic acid (94:6:0.2, vol/ $/ \mathrm{vol} / \mathrm{vol}$ ) (37). Plates were charred in a temperature-programmed oven after dipping in $10 \%$ cupric sulfate in $8 \%$ phosphoric acid (38). Quantitative spectrodensitometry was performed using a Shimadzu CS-910 Dual-Wavelength TLC scanner (Shimadzu Scientific Instruments, Inc., Columbia, MD) using a linear scanning mode (39). Peak areas were automatically integrated and lipid concentrations were calculated from standard curves fitted by least squares regression from a range of standards run each day of analysis.
Individual neutral lipid classes (TG, $\alpha, \alpha$ and $\alpha, \beta$-diglyceride (DG), $\alpha$ and $\beta-M G$ and FFA) were separated for subsequent fatty acid analysis as follows. Aliquots of the initial chloroform extract containing approximately $40 \mathrm{mg}$ lipid were spotted on predeveloped activated $\left(1 \mathrm{~h}\right.$ at $\left.100^{\circ} \mathrm{C}\right)$ preparative TLC plates. Plates were $20 \times 20 \mathrm{~cm}$ glass, coated with silica gel G-60 (E. Merck, Elmsford, NY) at $0.5 \mathrm{~mm}$ thickness. A standard lane was spotted on each plate with a mixture of neutral lipid standards. Plates were developed in the same 2-solvent system described above. Lipid classes were visualized and marked under UV light after spraying lightly with $0.2 \% 2^{\prime}, 7^{\prime}$-dichlorofluoresceine in absolute ethanol $(w / v)$. Silica gel areas containing the different classes were carefully scraped onto aluminum foil using a teflon spatula and transferred into separate glass tubes for subsequent transesterification.

Positional analysis. After initial comparison of results obtained from methods outlined by Brockerhoff (40) and Christie (41), the following procedure was used to hydrolyze TG to $\alpha, \alpha-\mathrm{DG}, \alpha, \beta-\mathrm{DG}, \alpha-\mathrm{MG}$ and $\beta$-MG. Anhydrous diethyl ether $(5 \mathrm{~mL})$ was added to approximately $50 \mathrm{mg}$ of mammary milk TG and the sample was set in an ice bath. To this was added $0.5 \mathrm{~mL}$ of ethyl magnesium bromide (Grignard reagent) (3.12 $\mathrm{M}$ in diethyl ether, Alfa Chemicals, Morton-Thiokol Inc., Davers, ME). The mixture was then agitated for $4 \mathrm{~min}$, after which $0.2 \mathrm{~mL}$ glacial acetic acid and $1 \mathrm{~mL} \mathrm{H}_{2} \mathrm{O}$ were added to stop the reaction, followed by $1 \mathrm{~mL}$ aqueous potassium bicarbonate $(2 \%)$. The tube was shaken, centrifuged, and the lower layer $\left(\mathrm{H}_{2} \mathrm{O}\right)$ pipetted out. The remaining ether layer was washed an additional three times with $\mathrm{H}_{2} \mathrm{O}$, removing as much $\mathrm{H}_{2} \mathrm{O}$ as possible at each wash. Anhydrous sodium sulfate was then added to dry the ether extract. Hydrolysis products were separated by TLC on silica gel (G-60) impregnated with $5 \%(\mathrm{w} / \mathrm{w})$ boric acid and developed in chloroform/acetone (96:4, vol/vol) (42). Fractions were visualized and isolated as described above for subsequent transesterification.

Preparation of fatty acid methyl esters. Transesterification and subsequent extraction of methyl esters were performed in the presence of the silica after initial verification that the presence or absence of silica did not alter fatty acid patterns. To each sample was added $1.5 \mathrm{~mL}$ of methylene chloride with $0.01 \%$ BHT and $3.0 \mathrm{~mL}$ Hilditch reagent $\left(0.5 \mathrm{~N} \mathrm{H}_{2} \mathrm{SO}_{4}\right.$ in methanol); the sample was flushed with nitrogen and stored in a glass tube with teflon-lined cap in the dark at room temperature for $72 \mathrm{~h}$. This method resulted in similar recoveries of methyl esters as were obtained using methanolic $\mathrm{HCl}$ at high temperatures, and was considered preferable due to the elimination of the risks of oven heating effects on PUFA, as well as the absence of artifact production. Alkali isomerizarion is reported with basic reagents (41).

Following transesterification, methyl esters were extracted into hexane. Hexane extracts were then concentrated to about $0.25 \mathrm{~mL}$ under nitrogen at $30^{\circ} \mathrm{C}$, applied to thin-layer plates and purified by preparative TLC using a solvent system of light petroleum ether $\left(30-60^{\circ} \mathrm{C}\right.$ boiling point)/diethyl ether $(95: 5, \mathrm{vol} / \mathrm{vol})$ (43). Methyl esters were visualized and collected from the silica gel by eluting with five column volumes of methylene chloride in a glass column plugged with glass wool. The sample was then concentrated under nitrogen, and isooctane was added at approximately $25 \mathrm{mg} \mathrm{lipid} / \mathrm{mL}$ isooctane. 
Gas-liquid chromatography. Fatty acid analyses were performed by temperature-programmed gas-liquid chromatography (GLC) on a Hewlett-Packard (Avondale, PA) Model 5880 Gas Chromatograph (GC) fitted with a Monarch series flexible glass capillary column $(25 \mathrm{~m} \times$ $0.25 \mathrm{~mm}$ i.d.) according to the methods of Sampugna $\mathrm{et} \mathrm{al}$. (43). The column was coated with SP-2340 (Quadrex Corp., New Haven, CT) at $25 \mu$ film thickness. The carrier gas was helium and the make up gas was nitrogen. The GC was equipped with a flame ionization detector (temperature $275^{\circ} \mathrm{C}$ ). The temperature of the injection port was maintained at $250^{\circ} \mathrm{C}$. Following sample injection, the temperature of the column was held at $153^{\circ} \mathrm{C}$ for $2.0 \mathrm{~min}$, then programmed to increase to $174^{\circ} \mathrm{C}$ at the rate of $2.25^{\circ} \mathrm{C}$ per min, then to increase to $200^{\circ} \mathrm{C}$ at the rate of $2.5^{\circ} \mathrm{C}$ per min and held there until the end of the run. The entire run time was approximately $24 \mathrm{~min}$.

Identifications of fatty acid methyl ester peaks were based on retention times of known standard mixtures run daily and also after isolation of fatty acid classes in selected samples from each species using silver nitrate ("argentation") chromatography (28). Methods for silver nitrate chromatography essentially followed recommendations by Christie (41), except that a mobile phase of hexane/diethyl ether $(90: 10$, vol/vol) was used to separate methyl esters containing zero to two double bonds, hexaneidiethyl ether $(40: 60$, vol/vol) to separate three to six double bonds and, finally, petroleum ether/diethyl ether/ acetic acid (90:8:2, vol/vol/vol) to separate zero to six double bonds for comparison. Peak areas were measured with an electronic integrator and normalized by individual response factors obtained from available standards. Sample injection volume was usually $0.3 \mu \mathrm{L}$, or about $7.5 \mu \mathrm{g}$ lipid. Fatty acids are designated by shorthand nomenclature of chain length:number of double bonds with $(n-x)$ denoting the position $(x)$ of the last double bond relative to the terminal methyl end.

Calculations and statistical analyses. The relative weight contribution (i.e., molecular weight) of individual fatty acid methyl esters differs greatly according to chain length and degree of unsaturation. Therefore, in the comparison of hydrolysis products to that of TG and in the positional analyses, fatty acid composition was expressed on a mol $\%$ basis rather than wt \%, unless otherwise indicated. The average molecular weight used in calculations for each component (TG, DG, MG and FFA) was based on their actual fatty acid compositions as determined from GLC analysis. The extent of hydrolysis in gastric samples was defined as the disappearance of TG (or increase in sum of hydrolysis products) from that originally present in milk.

Although 40-50 fatty acids (including isomers) were routinely observed in samples, fatty acid profiles were summarized according to the 14 major representative fatty acids of marine oils and seal lipids $(28,44)$. Because all isomers of a given monounsaturated component behaved identically in this study, isomers were combined and expressed as a single value.

The mol \% composition of the $s n-2$ position of milk TG was calculated from Grignard hydrolysis data as:

$$
[s n-2]=4[\alpha, \beta-\mathrm{DG}]-3[\mathrm{TG}]
$$

(e.g., refs. 40,41). For each fatty acid, the percent of the total amount present in milk which was located at the $s n-2$ position was then:

$$
\% @ s n-2=\frac{[s n-2]}{3[\mathrm{TG}]} \times 100
$$

The average composition of the $\alpha$-positions ( $s n-1, s n-3)$ was calculated as:

$$
[s n-1, s n-3]=\frac{3[\mathrm{TG}]-[s n-2]}{2}
$$

Calculations were performed using values obtained for $\alpha, \beta$ DG rather than for $\alpha, \alpha-D G$ since the latter tend to be more highly contaminated by acyl migration of $\alpha, \beta$-DG components (40).

Data are presented as mean \pm SEM. Unless otherwise indicated, all percentage values are mol \%. Statistical analyses were performed using Statview $512^{+}$(BrainPower Inc., Calabasas, CA) for the Macintosh.

\section{RESULTS}

Neutral lipid class composition and evidence of gastric hydrolysis. Milk TG composition and extent of gastric lipolysis were determined for each species (Table 1). Milk lipids of harp and hooded seals, stored in solvents from the time of collection, were composed of greater than $99 \%$ TG, with very minor amounts of FFA $(0.0-0.1 \%)$, DG

TABLE 1

Gastric Hydrolysis of Milk Lipid in Seal Neonates $a$

\begin{tabular}{lcccccc}
\hline & $\begin{array}{c}\text { Milk fat } \\
\text { (wt \%) }\end{array}$ & $\begin{array}{c}\text { TG in milk } \\
\text { (mol \%) }\end{array}$ & n & \multicolumn{3}{c}{ Gastric hydrolysis (mol \%) } \\
\cline { 5 - 7 } & Average $^{b}$ & Maximum & n \\
\hline Sea lion & $31.7^{c}$ & $98.4 \pm 0.06$ & $(4)$ & $9.3 \pm 1.33$ & $25 \%$ & $(20)$ \\
Harp seal & $51.8^{d}$ & $99.6 \pm 0.05$ & $(5)$ & $10.1 \pm 2.65$ & $37 \%$ & $(15)$ \\
Hooded seal & $61.0^{e}$ & $99.4 \pm 0.95$ & $(5)$ & $23.0 \pm 3.24$ & $56 \%$ & $(17)$ \\
\hline
\end{tabular}

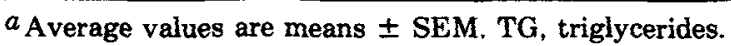

${ }^{b}$ Average values taken from mid-sampling point in sea lions $(28)$ and from all animals sampled in harp and hooded seals.

$c$ From ref. 31 .

$d$ From ref. 30.

$e$ From ref. 45.
} 
$(0.2-0.5 \%)$ and cholesterol (0.1-0.2\%). Sea lion milks $(\mathrm{n}=4$ samples which had not undergone thawing and refreezing), although stored frozen for six years without solvents prior to analysis, showed similarly high levels of TG (98.4\%). Although amounts of other components were sightly higher $(0.2 \%$ FFA, $0.8 \%$ DG and $0.1 \% \mathrm{MG})$, evidence of hydrolysis during storage was minimal. Cholesterol ester was not detected in any sample.

The comparison of neutral lipid class composition of gastric samples from pups revealed that substantial amounts of hydrolysis occurred in all species (Table 1). The average level of hydrolysis was at least twofold higher in the hooded seal than in the harp seal and sea lion and corresponded to the higher levels of total milk fat. Milk TG levels were found to drop as low as $43.6 \%, 62.8 \%$ and $75.0 \%$ in the hooded seal, harp seal and sea lion, respectively. In all cases, the primary products of hydrolysis were $\alpha, \beta$-DG $(0.2-20.0 \%)$ and FFA $(0.0-31.2 \%)$. Small amounts of MG $(0.0-4.4 \%)$ and only trace amounts of $\alpha, \alpha-\mathrm{DG}$ (0.0-1.1\% in harp and hooded seals only) were occasionally present.

Positional analysis. Positional analysis by Grignard hydrolysis of milk TG of all three species revealed distinct differences in the distributions of saturated (SFA), monounsaturated (MUFA) and polyunsaturated fatty acids (PUFA) as groups (Fig. 1). SFA tended to be very enriched at the $s n-2$ position of the milk TG, which contained essentially $70-90 \%$ of all SFA occurring in milks (see equation 2). By contrast, MUFA and PUFA were reduced at the $s n-2$ (Fig. 1), indicating their tendency to be esterified to the $\alpha$-position. In all three species, $13-27 \%$ of the MUFA in milk were at the $s n-2$ position, while only $2-11 \%$ of all PUFA were at $s n-2$.

Grignard hydrolysis data also provided information on the composition of individual fatty acids at the $s n-2$ position in comparison to that of the intact milk TG (Fig. 2). Specifically, the major fatty acids enriched at the middle position in seal milk TG were the shorter-chain saturates 14:0 and 16:0. The other principal SFA, 18:0, was enriched at $s n-2$ in the hooded seal but reduced at $s n-2$ in the harp seal and sea lion (not illustrated); however, it was less than $2 \%$ of total fatty acids and therefore absolute differences were not large. The other major fatty acid which was always enriched at $s n-2$ was $16: 1$.

The MUFA 18:1 was reduced at $s n-2$ in comparison to the intact TG, but absolute levels at $s n-2$ were still high; thus 16:1 and 18:1 contributed to most of the measurable MUFA at $s n-2$ (Fig. 1). The other MUFA, 20:1 and 22:1, as well as all major PUFA, were virtually absent from the $s n-2$ position (Fig. 2), indicating that all were located at the $\alpha$-position of the TG. Of the 14 major marine oil fatty acids, the PUFA 18:2n-6, 18:3n-3, 18:4n-3 and 20:4n-6 were variably reduced (or enriched) at $s n-2$, but are not illustrated in Figure 2 as these were generally less than $1.5 \%$ of total fatty acids and, therefore, absolute amounts and differences were very small. All three species exhibited the same patterns.

Fatty acid composition of milk TG and gastric hydrolysis products. Five mother/pup pairs were chosen for this part of the study to account for the variability in fatty acid compositions found among individual milks. Because some of the gastric samples from pups of each species had very low levels of hydrolysis, it was not always possible to obtain individual lipid classes (FFA, DG, MG) in

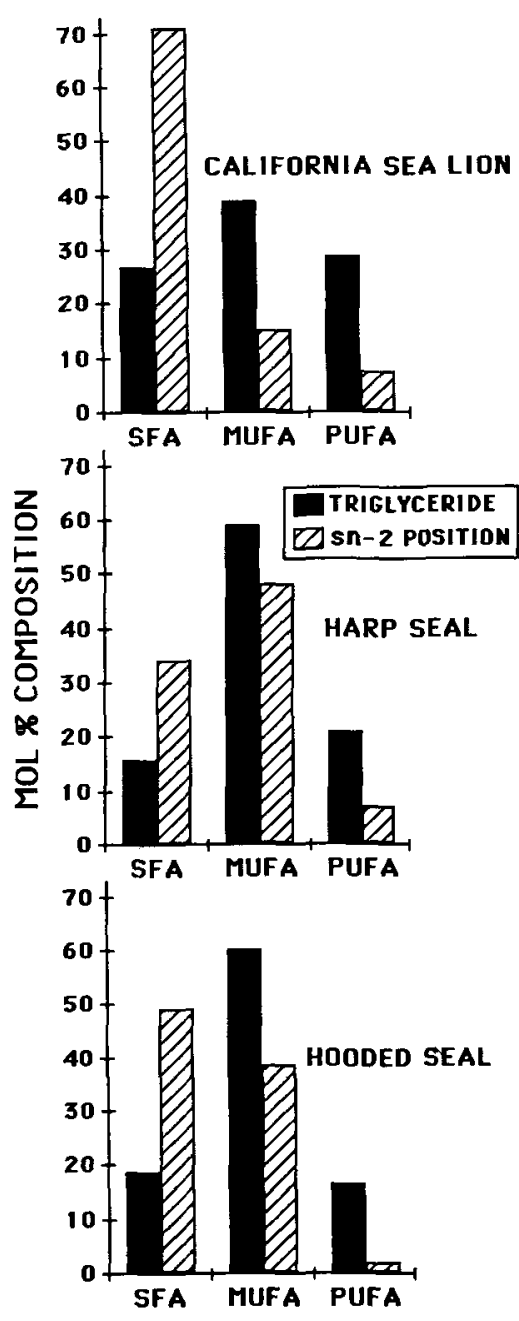

FIG. 1. Positional distribution of saturated (SFA), monounsaturated (MUFA) and polyunsaturated fatty acids (PUFA) in the triglyceride and at the $s n-2$ position in milk of three seal species as determined from Grignard hydrolysis. Mol \% composition of fatty acid groups at $s n-2$ position was calculated as: $[s n-2]=4[\alpha, \beta-D G]-3$ [TG].

sufficient quantity for fatty acid analysis of each class. Hence $\mathbf{n}$ differed from five in these cases, since composition of milk TG could only be compared to the FFA, DG and MG produced from the same milks after gastric digestion.

Analyses of the fatty acid composition of milk TG and the FFA released during gastric hydrolysis revealed highly significant differences between all components in each species $(P<0.01$, paired-t, Fig. 3$)$. The FFA were significantly reduced in the SFA 14:0 and 16:0, as well as in the MUFA 18:1, 20:1 and 22:1. In fact, the fatty acids preferentially released during gastric digestion, other than 18:0 and 16:1, were the long-chain PUFA, as determined by their significant enrichment in FFA. The n-3 PUFA 20:5n-3 was extraordinarily enriched, at up to five times the level in the TG. The single exception was 22:6n-3, which was surprisingly greatly reduced in FFA of all species (Fig. 3), at levels as low as one-sixth of that found in TG.

In examination of the composition of the other gastric hydrolysis products, as expected, the specific fatty acids 


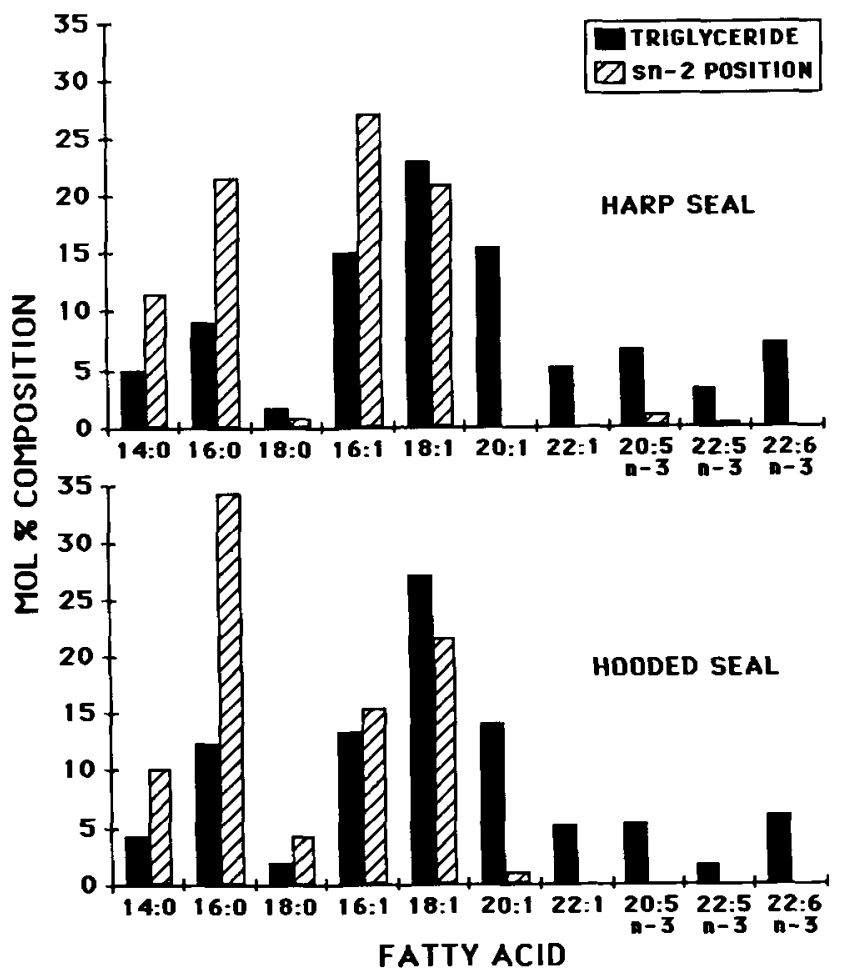

FIG. 2. Fatty acid composition of the triglyceride and at the $8 n-2$ position in seal milk as determined from Grignard hydrolysis. Mol \% composition of individual fatty acids at $8 n-2$ position was calculated as: $[s n-2]=4[\alpha, \beta-D G]-3$ [TG]. Of the 14 major marine oil fatty acids, the PUFA 18:2n-6, 18:3n-3, 18:4n-3 and 20:4n-6 are not illustrated as these were usually less than $1 \%$ of total fatty acids. Data for the sea lion are not presented but exhibited the same patterns.

which were reduced in FFA were found enriched in the DG compared to the TG. Likewise, those fatty acids found at enriched levels in FFA were found at reduced levels in DG. In other words, the fatty acids which were preferentially released from the TG consequently left the DG reduced (e.g., 20:5n-3 and 22:5n-3, Fig. 4). However, once again the single exception was $22: 6 n-3$, which was reduced in DG (indicating its release from the TG), but also greatly reduced in the FFA (Fig. 4). In spite of differences in absolute levels of hydrolysis and fatty acid compositions, the exact same patterns occurred in all three species.

The levels of individual fatty acids in the MG obtained from gastric hydrolysis accentuated the trends found in the DG in that the fatty acids enriched in DG as compared to TG were further enriched in MG and those reduced in DG were further reduced in MG. The MG obtained in gastric samples was almost exclusively $s n-2 \mathrm{MG}$, whereas Grignard hydrolysis produced both sn-2-MG and $\alpha-M G$, which were difficult to separate on preparative thinlayer plates and also likely to be cross-contaminated by acyl migration. Thus the gastric MG were considered to be truly representative of the $s n-2$ position. Hence, these data were compared to both the estimated $s n-2$ position, as determined from Grignard hydrolysis, as well as to the average composition of the $\alpha$-position of the milk TG (Fig. 5). In fact, in all species, the fatty acid composition of the MG obtained following gastric hydrolysis in vivo
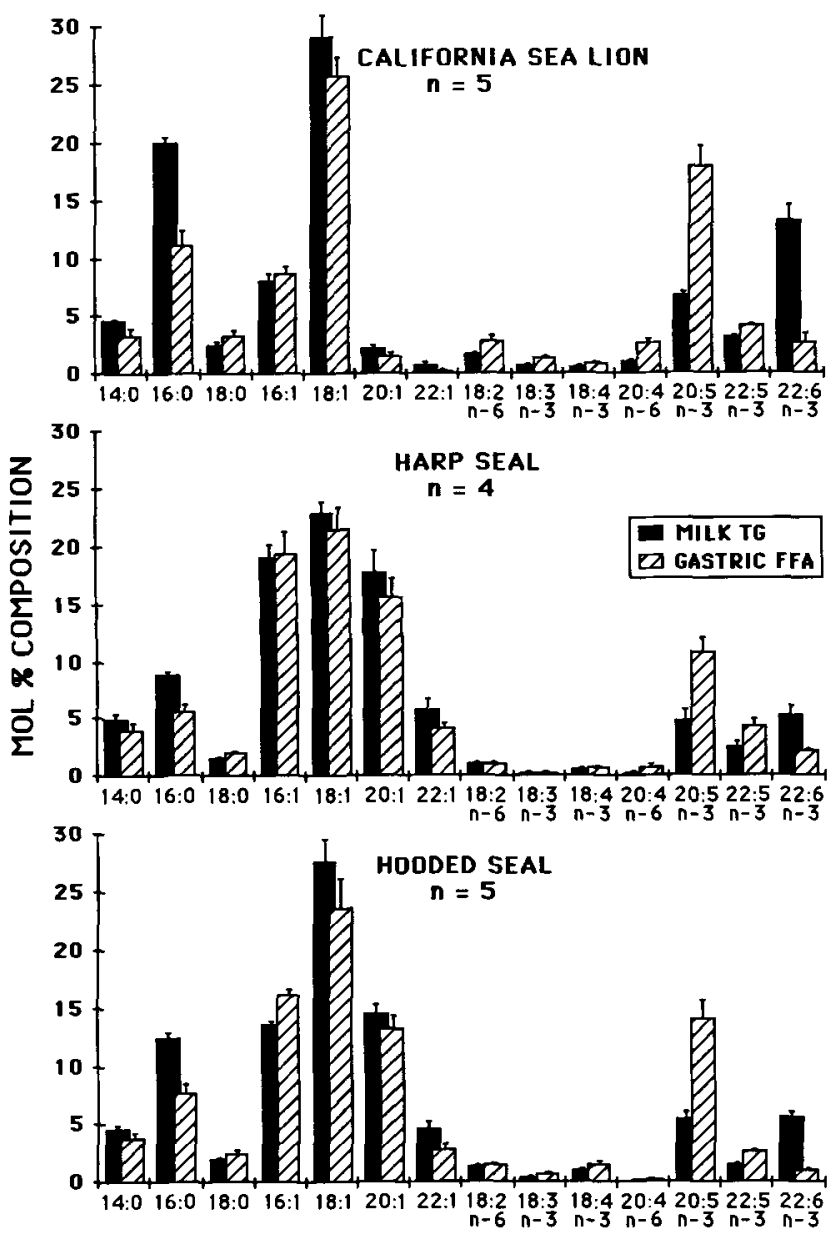

FATTY ACID

FIG. 3. Fatty acid composition of maternal milk TG and the FFA subsequently released during intragastric hydrolysis in pups of three seal species. Bars are mean mol \% composition and vertical lines are + 1 SEM. Differences in fatty acid composition between TG and gastric FFA were in the same direction for all species and were significant for all fatty acids $(P<0.01, n=14$, paired-t).

was remarkably similar to the estimated $s n$-2 composition calculated from the positional analysis using Grignard $\alpha, \beta$ DG. Conversely, the $\alpha$-position was in marked contrast to both estimates of the 2-position; in comparison, the $\alpha$ position was particularly reduced in 14:0, 16:0 and $16: 1$ and highly enriched in the MUFA, 20:1 and 22:1, and the n-3 PUFA (Fig. 5).

\section{DISCUSSION}

Milk lipid is the principal source of energy for suckling neonates of most mammalian species. Even in human milk at about $4 \%$ fat, it provides greater than $50 \%$ of total calories. More significantly, it is the sole source of essential and n-3 PUFA for the rapidly developing suckling neonate. Recent studies have shown that when maternal dietary intakes of long-chain PUFA are elevated, milk lipids become readily enriched in these fatty acids $(8,9,46)$. However, the actual absorption and utilization of these fatty acids by the neonate depends upon initial hydrolysis. 


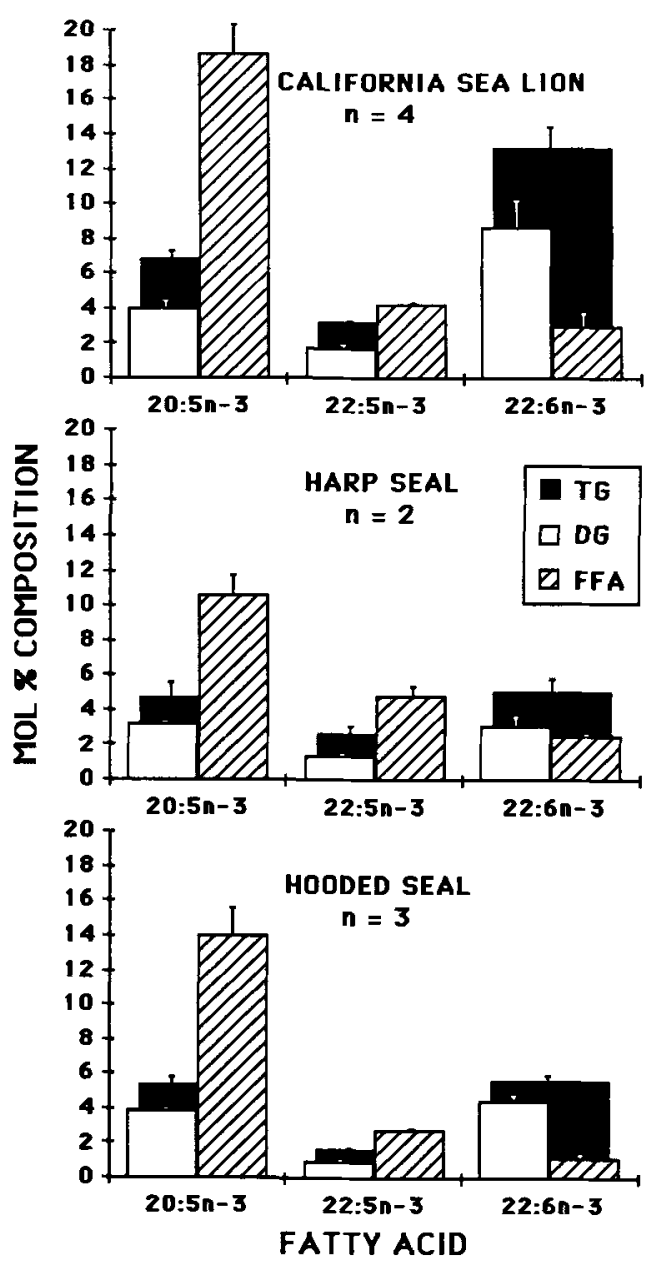

FIG. 4. Composition of the long-chain n-3 PUFA in maternal milk TG and in the hydrolysis products (DG and FFA) released during intragastric hydrolysis in pups of three seal species. Bars are mean mol \% composition and vertical lines are +1 SEM.

The importance of initial gastric digestion of dietary lipid by lingual and/or gastric lipases, especially in the neonate, has become increasingly evident (47). However, most information on hydrolysis has been limited to artificial homogeneous TG containing fatty acids of chain-length and degree of unsaturation of $18: 1$ or lower. Neonates of the three species of seals used in this study are similar to humans, some carnivores and the rabbit (48-52), in that they possess gastric lipase as the primary preduodenal lipase acting in the stomach $(28,34)$. The study of gastric hydrolysis of lipid-rich seal milks (Table 1), which contain high proportions of long-chain PUFA, may provide a better understanding of the digestion of the n-3 PUFA in milks in general and of the stereospecificity of gastric lipases.

Lipases typically exhibit specificity for substrate, position and/or fatty acid. Gastric and lingual lipases, which are analogous in both structure and function (53-57), are reported to exhibit considerable stereospecificity for the $\alpha$-position of the TG and to hydrolyze the $s n-3$ position twice as fast as the $s n-1$ position $(23,58-61)$. In this study, the primary products of hydrolysis were $\alpha, \beta$-DG and FFA in all three seal species. The occurrence of $\alpha, \beta-D G$ as

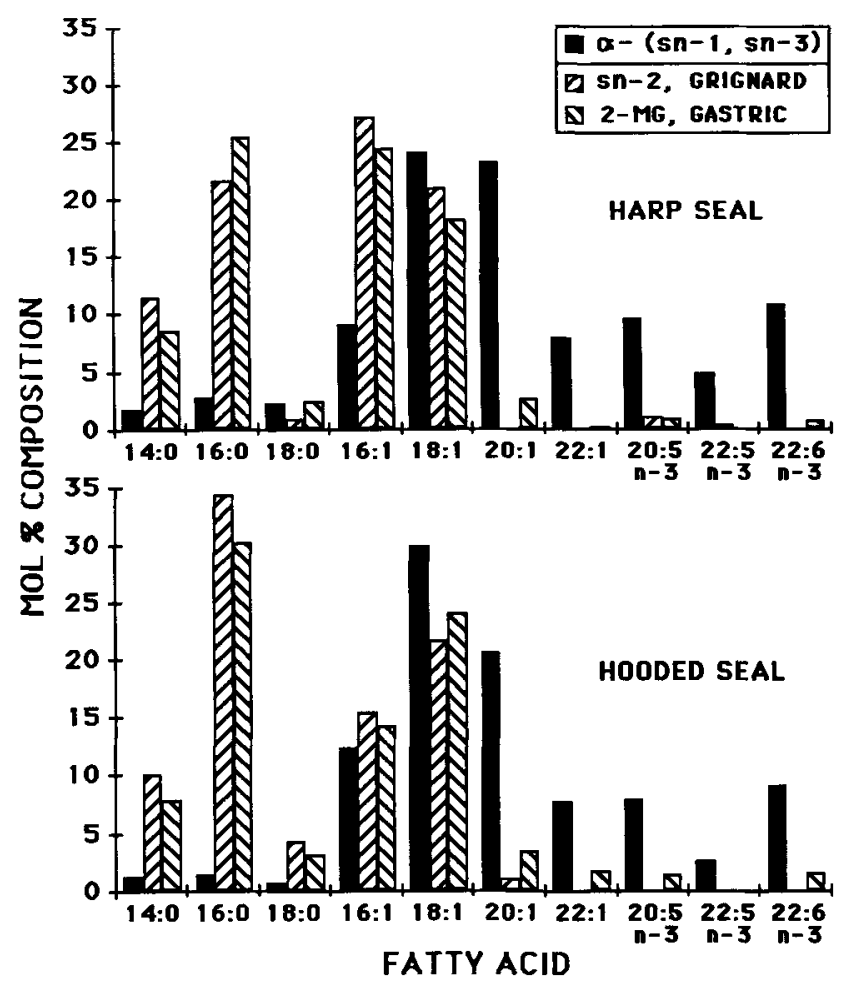

FIG. 5. The average fatty acid composition of the $\alpha$-positions ( $8 n-1$, $8 n-3)$ (from positional analysis), in comparison to the estimated ("Grignard") $8 n-2$ position (from positional analysis) and the $8 n-2$ MG obtained following intragastric hydrolysis. Average mol \% composition of the $\alpha$-positions was calculated as: $[s n-1, s n-3]=(3$ [TG] $[8 n-2]) / 2$. Mol \% composition of the $8 n-2$ position (Grignard) was calculated as: $[s n-2]=4[\alpha, \beta$-DG] -3 [TG]. Data for the sea lion are not presented but exhibited the same patterns.

virtually the sole DG product and the predominance of DG rather than MG as gastric hydrolysis products is consistent with the stereospecificity of gastric lipase. The occurrence of trace amounts of $\alpha, \alpha-\mathrm{DG}$ was probably due to small amounts of acyl migration. The $s n-2$ position did not appear to be hydrolyzed.

Thus, stereospecific analysis of TG and hydrolysis products provides some clues about the mechanisms of gastric digestion. Although pancreatic lipase hydrolysis is often used for positional analyses, it is not appropriate for TG which contain long-chain PUFA in the primary positions since pancreatic lipase is specific for the $\alpha$-position, but does not hydrolyze long-chain PUFA, such as 20:5n-3, $22: 5 n-3$ and $22: 6 n-3(13,14,41)$. In contrast, Grignard reagent (ethyl magnesium bromide) releases the fatty acids (as tertiary alcohols) from all three positions of TG, generating intermediate products, which include $\alpha, \beta$ - and $\alpha, \alpha$ DG. In this study, Grignard hydrolysis tended to produce some breakdown products within classes which were recorded as unidentified peaks during GLC analysis and excluded from calculations. The cleanest products were the $\alpha, \beta$-DG. Because $\alpha, \beta$-DG produced during chemical hydrol$y$ sis also tend to be free of acyl migration (41), these were used for calculations of the 2-position.

Previous studies have shown that the positional distribution of specific fatty acids in milk TG tend to be very similar across a wide range of mammalian species $(7,40$, 


\section{S.J. IVERSON $E T A L$.}

62,63); however, the distribution of long-chain and n-3 PUFA in milks has rarely been studied. The positional data obtained in this study are comparable to those found in other marine mammal species (Table 2). In all species, the $s n-2$ position of milk TG contained about $70-100 \%$ of the 14:0 and 16:0 present in milk (Table 2 and Fig. 2), a finding characteristic of the milks of many mammalian species, including human, pig and $\operatorname{dog}(7,62,63)$. This distribution is consistent with the observed action of gastric lipase in seal neonates. Preferential hydrolysis of the $\alpha$-position by gastric lipase would explain the paucity of 14:0 and 16:0 in FFA (Fig. 3) and the relative accumulation of these fatty acids in DG. Similar accumulations of 14:0 and 16:0 have been reported in rat milk DG and MG following gastric hydrolysis (66).

In all mammalian species previously investigated, 18:0 has been found selectively esterified at the $s n-1$ position with roughly $9-11 \%$ occurring at the $s n-2(62,63)$. Similar proportions of 18:0 have been found at the $s n-2$ in most seal and whale milks (Table 2), thus the slight enrichment of 18:0 in gastric FFA in this study (Fig. 3) may have been due to accumulation from hydrolysis of both $s n-1$ and $s n-3$. However, the hooded seal is exceptional in that selective esterification (74\%) appears to occur at $s n$-2 (Table 2), suggesting that some aspects of milk TG structure may actually differ among species, as has previously been indicated in selected species (63).

In marine mammals, as in virtually all other species studied $(62,63), 16: 1$ tends to be evenly distributed at all positions of the milk TG, whereas 18:1 tends to be more enriched at the $\alpha$-positions. As expected, $16: 1$ and 18:1 are readily hydrolyzed during gastric lipase action. In the Weddell seal, 16:1 is more prevalent at $s n-3$ than at $s n-1$, whereas the reverse is true for 18:1 (63), which may account for the slight enrichment and reduction in FFA of 16:1 and 18:1, respectively, as compared to milk TG in this study (Fig. 3). MUFA 20:1 and 22:1, which are found at unusually high levels in some marine oils and milks (e.g., Fig. 3), were virtually absent from the $s n-2$ position of milks of all marine species studied (Fig. 2 and Table 2) and thus primarily at the $\alpha$-position (Fig. 5). In human milk, 20:1 has been reported to be preferentially located at $s n-1$ (7); locations of $20: 1$ and 22:1 have not been reported for the milks of other species. Reasonably high levels of release during gastric hydrolysis, yet at slightly reduced levels in FFA compared to TG (Fig. 3), suggest their tendency also to be esterified to the $s n-1$ position in seal milk.

While the $s n-2$ position of pinniped milk TG was found to contain variable and sometimes relatively high proportions of the minor PUFA 18:2n-6, 18:3n-3, 18:4n-3 and 20:4n-6 (Table 2), these fatty acids usually accounted for less than $1 \%$ of fatty acids. Data from other species in. dicate variable distribution of $18: 2 n-6$ and $18: 3 n-3$, but the majority of both tend to be found at the $s n-3$ position (63). The most important long-chain n-3 PUFA of seal milk, comprising up to $26 \%$ of all fatty acids (28), are $20: 5 n-3$, 22:5n-3 and 22:6n-3. These fatty acids were found to be essentially absent from the $s n-2$ position in all species (Fig. 2 and Table 2) and are most likely located primarily at the $s n-3$ position $(40,63)$. The slightly higher levels of these PUFA reported at the $s n-2$ position in the milks of the fur seal, elephant seal and fin whale $(64,65)$ may have resulted from the use of pancreatic lipase rather than Grignard hydrolysis for positional analysis. Thus, the fatty acids most preferentially released from the milk TG during gastric digestion (other than 16:1 and 18:0) were the PUFA of chain-length 18 carbons or longer (Fig. 3), which is consistent with their location in the milk TG leg., Fig. 5) and the specificity of gastric lipase for the $s n-3$ position. These findings were supported by the relative depletion of the long-chain PUFA in the DG fraction (Fig. 4). Preferential release during intragastric hydrolysis has also been reported in puppies for the major PUFA of dog milk, 18:2n-6, 18:3n-3 and 20:4n-6 (47).

The single PUFA which did not fit this pattern was $22: 6 n-3$. While the extraordinary enrichment of $20: 5 n-3$ in FFA during gastric hydrolysis was surprising (Figs. 3 and 4), the extreme reduction of $22: 6 n-3$ in FFA was even more surprising since it is likely that $22: 6 \mathrm{n}-3$ also occurs

TABLE 2

Comparison of Percentage Distribution of Fatty Acids at the sn-2 Position in Milk TG of Various Marine Mammals ${ }^{a}$

\begin{tabular}{|c|c|c|c|c|c|c|c|c|c|c|c|c|c|c|}
\hline & $14: 0$ & $16: 0$ & $18: 0$ & $16: 1$ & $18: 1$ & $20: 1$ & $22: 1$ & $\begin{array}{c}18: 2 \\
\mathrm{n}-6\end{array}$ & $\begin{array}{c}18: 3 \\
n-3\end{array}$ & $\begin{array}{c}18: 4 \\
n-3\end{array}$ & $\begin{array}{c}20: 4 \\
n-6\end{array}$ & $\begin{array}{c}20: 5 \\
n-3\end{array}$ & $\begin{array}{c}22: 5 \\
n-3\end{array}$ & $\begin{array}{l}22: 6 \\
n-3\end{array}$ \\
\hline Range in milks $b$ & $2-11$ & $9-23$ & $1-4$ & $4-17$ & $19-39$ & $2-17$ & $0.3-7$ & $1-2$ & $0.3-1$ & $0-1$ & $0.3-1$ & $1-14$ & $1-4$ & $4-16$ \\
\hline CA sea lion $c$ & 100 & 90 & 0 & 50 & 0 & 0 & 0 & 48 & 52 & 62 & 0 & 4 & 4 & 0 \\
\hline Antarctic fur seald & 63 & 79 & 0 & 37 & 13 & 0 & 0 & - & - & - & - & 7 & 0 & 0 \\
\hline Harp sealc & 76 & 79 & 17 & 60 & 30 & 0 & 0 & 59 & 44 & 64 & 11 & 6 & 5 & 0 \\
\hline Hooded sealc & 79 & 92 & 74 & 38 & 27 & 3 & 1 & 49 & 6 & 0 & 0 & 0 & 0 & 0 \\
\hline N. elephant seald & 78 & 92 & 20 & 57 & 26 & 6 & 7 & - & - & - & - & 13 & 3 & 16 \\
\hline Weddell seale & 68 & 69 & 11 & 41 & 16 & - & - & 37 & 33 & -..... & $\ldots$ & -2 & & \\
\hline Fin whale $f$ & 84 & 80 & 7 & 57 & 12 & 2 & 1 & 18 & 31 & 45 & 11 & 8 & 4 & 6 \\
\hline
\end{tabular}

$a$ Percent of each fatty acid present in milk which is located at the $s n-2$ position of the triglyceride (TG) as determined from positional analyses; dashes indicate values not reported or analyzed. \%@sn-2 $=[s n-2] / 3[\mathrm{TG}] \times 100$. CA, California.

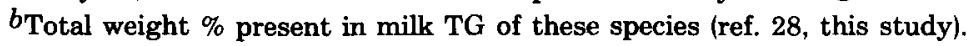

$c$ From this study.

$d$ Calculated from ref. 64 .

$e$ Calculated from ref. 63. All PUFA $>18: 3$ reported as a single value.

$f$ Calculated from ref. 65 . 
primarily at the $s n-3$ position (this study, refs. 40 and 63). Additionally, like $20: 5 n-3$ and $22: 5 n-3$, proportions of 22:6n-3 were found to be depleted in the gastric DG obtained from all three species, suggesting that it also had been released from the TG (Fig. 4). In all samples, 22:6n-3 was stable in all fractions (total, TG, DG, FFA) and was readily and reproducibly collected from preparative plates, transesterified and measured. The lower levels of $22: 6 \mathrm{n}-3$ found in both the DG fraction (as expected) and FFA fraction were considered to be accurate measurements; no evidence of loss through autooxidation or overlap in various fractions was found, despite numerous efforts to elucidate such occurrences. The DG and FFA data, in addition to the residual TG available, also demonstrated no evidence of intermolecular specificity by lipases (i.e, preferential hydrolysis of TG species). Thus the extreme reduction of 22:6n-3 in FFA, in conjunction with the enrichment of 20:5n-3 in FFA at levels much higher than expected, suggests some kind of metabolic loss, conversion or preferential absorption. The retroconversion of $22: 6 n-3$ to $20: 5 n-3$ has previously been reported (67-69), although how this process could possibly occur during digestive metabolism and whether this could account for the observed discrepancies is unclear. Given that most hydrolyzed lipids are believed to remain with the parent milk fat globule until its disruption in the intestine $(23,24)$, preferential absorption of a single and very long-chain fatty acid, such as $22: 6 n-3$, seems unlikely. Clearly, these patterns require further investigation.

The rapidly accumulating information on the effects of n-3 PUFA in marine oils confirms the notion that these are important dietary components for both infants and adults (4-7). Nevertheless, this stands in contrast to the relatively limited knowledge about the digestion of $n-3$ PUFA, particularly from milk TG. In this study, high intakes of total milk fat and PUFA were coupled with high levels of gastric lipolysis in pups. Despite apparent individual and species variation in the extent of hydrolysis, the same fatty acids, primarily the long-chain and $n-3$ PUFA, were preferentially released in pups of all three species. This is explained by their unique location in the milk TG at the $\alpha$ - and probably mostly at the $s n$-3 position and by the corresponding specificity of gastric lipase. Reports of preferential hydrolysis of short- and mediumchain fatty acids in milk (e.g., ref. 66) may be partly attributable to the fact that these fatty acids, at least in the human and the rat, are also preferentially esterified at the $s n-3$ position in milk $(70,71)$.

We conclude that the n-3 PUFA in milk are efficiently hydrolyzed by gastric lipase and that this has important implications for the digestion of milks enriched in PUFA by neonates in general. Given the general similarity in milk TG structure among species and the fact that many mammalian species, including the human, possess lingual and/or gastric lipases, hydrolysis of milk fat enriched in the important long-chain n-3 PUFA may be primarily dependent upon the actions of these lipases prior to reaching the intestine. Furthermore, while pancreatic lipase may not be able to continue hydrolysis of these fatty acids in the $\alpha$-positions, bile salt-stimulated lipase, present in the milk of species such as seals, humans and a number of other primates and carnivores studied, non-specifically hydrolyzes TG and partial glycerides in the intestine (72-74), and may account for further PUFA release.

\section{ACKNOWLEDGMENTS}

We are very grateful to Dr. W. D. Bowen of the Department of Fisheries and Oceans, Canada, for organizing the expedition to the pack ice, for assistance in the collection of harp and hooded seal samples and for valuable comments on an earlier version of the manuscript. We thank members of the U.S. Navy at the Pacific Missile Test Center, Pt. Mugu, CA, for permission to conduct research on San Nicolas Island and for logistical support during field studies. We thank Dr. D. J. Boness for assistance in sample collection during both field studies and for general research support. We also thank Dr. J. Bitman and especially D. L. Wood of the USDA Milk Secretion and Mastitis Laboratory, Beltsville, MD, for their time, advice and use of their laboratory facilities to conduct TLC work. This study was supported by research grants (to S.J.I. and O.T.O.) from the Friends of the National Zoo and the Office of Fellowships and Grants of the Smithsonian Institution, Washington, D.C., and by the Department of Fisheries and Oceans, Canada.

\section{REFERENCES}

1. Phillipson, B.E., Rothrock, D.W., Connor, W.E., Harris, W.S., and Illingworth, D.R. (1985) N. Engl. J. Med. 312, 1210-1216.

2. Goodnight, S.H., Harris, W.S., Connor, W.E., and Illingworth, D.R. (1982) Arteriosclerosis 2, 87-113.

3. Kremer, J.M., Bigauoette, J., Michalek, A.V., Timchalk, M.A., Lininger, L., Rynes, R.I., Huyck, C., Zieminski, J., and Bartholomew, L.E. (1985) Lancet 1, 184-187.

4. Clandinin, M.R., Chappell, J.E., Heim, T., Swyer, P.R., and Chance, G.W. (1981) Early Human Develop. 5, 355-366.

5. Crawford, M.A., Hassam, A.G., and Rivers, J.P.W. (1981) Prog. Lipid Res. 20, 31-40.

6. Garcia, R.E., and Moodie, D.S. (1989) Pediatrics 84, 751-755.

7. Jensen, R.G. (1989) in Textbook of Gastroenterology and Nutrition in Infancy, 2nd edn. (Lebenthal, E., ed.) pp. 157-208, Raven Press, Ltd., New York.

8. Harris, W.S., Connor, W.E., and Lindsey, S. (1984) Am. J. Clin. Nutr. 40, 780-785.

9. Henderson, R.A., Jensen, R.G., Ferris, A.M., Lammi-Keefe, C.J., and Dardick, K.R. (1992) Lipids 27, 863-869.

10. Sunshine, P., Herbst, J.J., Koldovsky, O., and Kretchmer, N. (1971) Annals. N.Y. Acad. Sci. 176, 16-29.

11. Tavolini, N., and Schaffner, F. (1989) Biol. Neonate 55, 124-135.

12. Watkins, J.B. (1974) Pediat. Clin. N. Am. 21, 501-512.

13. Bottino, N.R., Vandenberg, G.A., and Reiser, R. (1967) Lipids 2, 489-493.

14. Brockerhoff, H. (1970) Biochim. Biophys. Acta 22, 559-565.

15. Ackman, R.G. (1988) Atherosclerosis 70, 171-173.

16. Brockerhoff, H., and Hoyle, R.J. (1963) Arch. Biochem. Biophys. $102,452-457$.

17. Brockerhoff, H., Hoyle, R.J., Hwang, P.C., and Litchfield, C. (1968) Lipids 3,24-29.

18. Litchfield, C. (1968) Lipids 3, 417-419.

19. Brockerhoff, H., Ackman, R.G., and Hoyle, R.J. (1963) Arch. Biochem. Biophys. 100, 9-12.

20. Brockerhoff, H., Hoyle, R.J., and Hwang, P.C. (1966) Can. J. Biochem. 44, 1519-1525.

21. Brockerhoff, H., Hoyle, R.J., and Hwang, P.C. (1967) Biochim. Biophys. Acta 144, 541-548.

22. Lawson, L.D., and Hughes, B.G. (1988) Biochem. Biophys. Res. Commun. 152, 328-332.

23. Patton, J.S., Rigler, M.W., Liao, T.H., Hamosh, P., and Hamosh, M. (1982) Biochim. Biophys. Acta 712, 400-407.

24. Patton, J.S., Vetter, R.D., Hamosh, M., Borgström, B., Lindstrom, M., and Carey, M.C. (1985) Food Microstruct. 4, 29-41.

25. Bitman, J., Wood, D.L., Hamosh, M., Hamosh, P., and Mehta, N. (1983) Am. J. Clin. Nutr. 38, 300-312.

26. Jensen, R.G. (1988) The Lipids of Human Milk, CRC Press, Boca Raton.

27. Smith, S., and Abraham, S. (1975) in Lipid Research (Paoletti, R., and Kritchevsky, D., eds.) Vol. 13, pp. 195-239, Academic Press, NY.

28. Iverson, S.J. (1988) Composition, Intake and Gastric Digestion of Milk Lipids in Pinnipeds, Ph.D. Thesis, University of Maryland, College Park. 


\section{S.J. IVERSON $E T A L$.}

29. Iverson, S.J., and Oftedal, O.T. (1989) Eighth Biennial Conference on the Biology of Marine Mammals, Monterey, CA, Dec. 1989, Abstracts.

30. Oftedal, O.T., Boness, D.J., and Tedman, R.A. (1987) Curr. Mammal 1, 175-245.

31. Oftedal, O.T., Iverson, S.J., and Boness, D.J. (1987) Physiol Zool $60,560-575$.

32. Bowen, W.D., Oftedal, O.T., and Boness, D.J. (1985) Can. J. Zool. 63, 2841-2846.

33. Bowen, W.D., Boness, D.J., and Oftedal, O.T. (1987) Can. J. Zool $65,1-8$.

34. York, C.M., Yaa. J., Hamosh, M., Oftedal, O.T., Freed, L.M., and Hamosh, P. (1987) FASEB J. 46, 1084 (Abstract).

35. Yurkowski, M., and Brockerhoff, H. (1966) Biochim. Biophys. Acta $125,55-59$.

36. Folch, J., Lees, M., and Sloane-Stanley, G.H. (1957) J. Biol Chem 226, 497-509.

37. Bitman, J., Wood, D.L., and Ruth, J.M. (1981) J. Liq. Chrom. 4, 1007-1021.

38. Bitman, J., and Wood, D.L. (1982) J. Liq. Chrom. 5, 1152-1162.

39. Bitman, J., and Wood, D.L. (1981) J. Liq. Chrom. 4, 1023-1034.

40. Brockerhoff, H. (1971) Lipids 6, 942-956.

41. Christie, W.W. (1982) Lipid Analysis, 2nd edn., Pergamon Press, Oxford.

42. Thomas III, A.E., Scharoun, J.E., and Ralston, H. (1964) J. Am. Oil Chem. Soc. 42, 789-792.

43. Sampugna, J., Pallansch, L.A., Enig, M.G., and Koeney, M. (1982) J. Chromatogr. 249, 245-255.

44. Ackman, R.G., Ratnayake, W.M.N., and Olsson, B. (1988) J. Am. Oil Chem. Soc. 65, 136-138.

45. Oftedal, O.T., Boness, D.J., and Bowen, W.D. (1988) Can. J. Zool $66,318-322$.

46. Iverson, S.J., and Oftedal, O.T. (1992) Lipids 27, 940-943.

47. Iverson, S.J., Kirk, C.K., Hamosh, M., and Newsome, J. (1991) Biochim. Biophys. Acta 1083, 109-119.

48. DeNigris, S.J., Hamosh, M., Kasbekar, D.K., Fink, C.S., Lee, T.C. and Hamosh, P. (1985) Biochim. Biophys. Acta 836, 67-72.

49. Fink, C.S., Hamosh, M., Hamosh, P., DeNigris, S.J., and Kasbekar, D.K. (1985) Am. J. Physiol 248, G68-G72.

50. DeNigris, S.J., Hamosh, M., Kasbekar, D.K., Lee T., and Hamosh, P. (1988) Biochim. Biophys. Acta 959, 38-45.

51. Moreau, H., Laugier, R., Gargouri, Y., Ferrato, F., and Verger, R. (1988) Gastroenterology 95, 1221-1226.

52. Kirk, C.L., Iverson, S.J., and Hamosh, M. (1991) Biol Neonate 59, 78-85.

53. Hamosh, M., and Hamosh, P. (1989) in Human Gastrointestinal Development (Lebenthal, E., ed.), pp. 251-276, Raven Press, New York.
54. Tirrupathi, C., and Balasubramanian, K.A. (1982) Biochim. Biophys. Acta 712, 692-697.

55. Bernback, S., Hernell, O., and Blackberg, L. (1985) Eur. $J$. Biochem. 148, 233-238.

56. Docherty, A.J.P., Bodmer, M.W., Angal, S., Verger, R., Riviere, C., Lowe, P.A., Lyons, A., Emtage, J.S., and Harris, T.J.R. (1985) Nucleic Acids Res. 13, 1891-1903.

57. Bodmer, M.W., Angal, S., Yarrington, G.T., Harris, T.J.R., Lyons, A., King, D.K., Pieroni, G., Riviere, C., Verger, R., and Lowe, P.A. (1987) Biochim. Biophys. Acta 909, 237-244.

58. Fernando-Warnakulasuriya, G.J.P., Staggers, J.E., Frost, S.C., and Wells, M.A. (1981) J. Lipid Res. 22, 668-674.

59. Staggers, J.E., Warnakulasuriya, G.J.P., and Wells, M.A. (1981) J. Lipid Res. 22, 675-679.

60. Paltauf, R., Esfandi, F., and Holasek, A. (1974) FEBS Lett. 40, 119-123.

61. Jensen, R.G., Dejong, F.A., Clark, R.M., Palmgren, L.G., Liaa, T.H., and Hamosh, M. (1982) Lipids 17, 570-572.

62. Davies, D.T., Holt, C., and Christie, W.W. (1983) in Biochemistry of Lactation (Mepham, T.B., ed.), pp. 71-117, Elsevier Science Publishers B.V., Amsterdam.

63. Parodi, P.W. (1982) Lipids 17, 437-442

64. Puppione, D.L., Jandacek, R.J., Kunitake, S.T., and Costa, D.P (1989) in Dietary $\omega 3$ and $\omega 6$ Fatty Acids (Galli, C., and Simopoulos, A.P., eds.), pp. 361-367, Plenum Press, New York.

65. Ackman, R.G., Eaton, C.A., and Hooper, S.N. (1968) Can. J. Biochem, 46, 197-203.

66. Bitman, J., Wood, D.L., Liao, T.H., Fink, C.S., Hamosh, P., and Hamosh, M. (1985) Biochim. Biophys. Acta 834, 58-64.

67. Schlenk, H., Sand, D.M., and Gellerman, J.L. (1969) Biochim. Biophys. Acta 187, 201-207.

68. Stoffel, W., Ecker, W., Assad, H., and Sprecher, H. (1970) Zeitschr. Physiol Chem. 351, 1545-1554.

69. von Schacky, C., and Weber, P.C. (1985) J. Clin. Invest. 76, $2446-2450$.

70. Breckenridge, W.C., Marai, L. and Kuksis, A. (1969) Can. J. Biochem. 47, 761-769.

71. Lin, C.Y., Smith, S. and Abraham, S. (1976) J. Lipid Res. 17 647-656.

72. Freudenberg, E. (1953) Die Frauenmilch-Lipase Karger, Basel.

73. Hernell, O., and Olivecrona, T. (1974) J. Lipid Res. 15, 367-372.

74. Freed, L.M., York, C.M., Hamosh, M., Sturman, J.T., Oftedal, O.T., and Hamosh, P. (1986) in Human Lactation Maternal Environmental Factors (Hamosh, M., and Goldman, A.S., eds.) Vol. 2, pp. 595-602, Plenum Press, New York.

[Received January 11, 1991, and in revised form July 22, 1991; Revision accepted December 21, 1991] 